\title{
Papel dos atributos dos profissionais médicos na produção da abordagem centrada na pessoa em atenção primária à saúde
}

\author{
Role of physicians' attributes in the production of the \\ person-centered approach in primary health care
}

Rodrigo Caprio Leite de Castro (https://orcid.org/0000-0002-6006-6787) ${ }^{1}$

Daniela Riva Knauth (https://orcid.org/0000-0002-8641-0240) ${ }^{1}$

${ }^{1}$ Programa de PósGraduação em

Epidemiologia, Faculdade de Medicina, Universidade Federal do Rio Grande do Sul. R. Ramiro Barcelos 2.400, $2^{\circ}$ andar. 90035-003. Porto Alegre RS Brasil. rcastro@hcpa.edu.br

\begin{abstract}
Even though several factors influence the medical approach, there is few research examining the association of characteristics at the doctor's level with the kind of practice developed by this provider. This study aims at establishing the factors that, at the doctor's level, are associated with the high degree of patient-centered clinical method (PCCM) orientation assigned by the cared persons to the approach of this provider in primary health care (PHC). This cross-sectional study was conducted with hypertensive or diabetic patients and doctors in the 12 health care centers of a PHC service in Porto Alegre (RS), Brazil. The "Patient Perception of Patient-Centeredness" (PPPC) tool was used to measure the degree of PCCM orientation. The variables concerning the doctor's age, gender, time since graduation, service and unit seniority, and participation in continuing education outside the studied service were correlated with the median of the PPPC general score estimated for each professional. We also found a significant difference in the comparison between the medians of the PPPC general score in each health care center. We discuss the influence of professional experience, gender, continuing education, and the context on the approach developed by the doctor.
\end{abstract}

Key words Patient-centered care, Quality of health care, Primary health care
Resumo Embora inúmeros fatores influenciem a abordagem médica, poucas pesquisas exploram a associação de características presentes no nível do médico com o tipo de prática por ele desempenhada. Este estudo tem como objetivo determinar os fatores que, no nivel do médico, associam-se ao alto grau de orientação ao método clínico centrado na pessoa (MCCP), atribuído, pelas pessoas atendidas, à abordagem desse profissional em atenção primária à saúde (APS). O delineamento é transversal, realizado com pacientes hipertensos elou diabéticos e médicos em 12 unidades de um serviço de APS em Porto Alegre, RS. Para se medir o grau de orientação ao MCCP, utilizouse o instrumento "Percepção do paciente sobre o centramento da consulta" (PPCC). As variáveis idade do médico, gênero, tempo de formado, de trabalho no serviço e na unidade e participação em educação continuada fora do serviço estudado foram correlacionadas com a mediana do escore geral do PPCC estimada para cada profissional. Verificou-se também diferença significativa na comparação entre as medianas do escore geral do PPCC de cada unidade de saúde. Discute-se a influência da experiência profissional, do gênero, da educação continuada e do contexto na abordagem desempenhada pelo médico.

Palavras-chave Assistência centrada no paciente, Qualidade da assistência à saúde, Atenção primária à saúde 


\section{Introdução}

A insuficiência da abordagem médica centrada na doença se faz notar particularmente no âmbito da atenção primária à saúde (APS), em que os problemas dos pacientes não se restringem ao aspecto físico, mas imbricados a questões psicológicas e sociais ${ }^{1}$. O processo de atenção na APS requer, ainda, o acompanhamento das pessoas ao longo do tempo, o manejo de uma variedade de problemas de saúde nas mesmas pessoas e a consideração do contexto delas e das famílias nesse manejo ${ }^{1,2}$.

É por essas razões que, no Brasil, a medicina centrada na pessoa (MCP), que propõe uma abordagem médica que vai além da doença, vem sendo introduzida e estudada, principalmente por médicos que atuam na atenção primária à saúde (APS), com especialidade em medicina de família e comunidade (MFC).

A fim de operacionalizar essa abordagem, Stewart e colaboradores ${ }^{3}$ propuseram o método clínico centrado na pessoa (MCCP), constituído por quatro componentes interativos: "explorando a saúde, a doença e a experiência da doença", "entendendo a pessoa como um todo", "elaborando um plano conjunto de manejo dos problemas" e "intensificando a relação entre pessoa e médico".

Os fatores que podem influenciar a abordagem médica são de diferentes tipos, indo desde os diretamente relacionados ao paciente e ao médico até os macrossociais, como aqueles de ordem socioeconômica e cultural, passando pelas características do próprio serviço de saúde no qual ocorre a interação médico-paciente e pelas políticas públicas. A complexidade aumenta ao considerarmos ainda as muitas interações entre esses fatores e suas potenciais influências na produção de cuidado ${ }^{4-6}$.

A respeito da atuação médica em APS, apesar do cenário favorável encontrado nesse nível de atenção para a aplicação da MCP, supõe-se que haja médicos de família e comunidade com abordagens que se situam ao longo de todo o continuum de foco existente entre o centramento na pessoa, de um lado, e na doença, de outro. Esse pressuposto é reforçado pelos estudos de Roter et al. ${ }^{7}$, Flocke, Miller et al. ${ }^{8}$ e Roy, Vanheule et al. ${ }^{9}$, que buscaram caracterizar as abordagens médicas e as suas diferenças entre os profissionais.

Roter et al. ${ }^{7}$, em pesquisa realizada em Baltimore, Estados Unidos, por meio da análise de consultas em APS registradas em áudio e de en- trevistas com pacientes e médicos após as consultas (537 pacientes e 127 médicos), mostraram cinco padrões de comunicação médico-paciente distintas: "estritamente biomédica", caracterizada por perguntas fechadas e conversa biomédica (32\%), "biomédica expandida", padrão restrito, mas com níveis moderados de discussão psicossocial (32\%), "biopsicossocial", refletindo um equilíbrio de temas biomédicos e psicossociais (20\%), "psicossocial", caracterizada pelo predomínio de assuntos psicossociais (8\%), e "consumista", caracterizada sobretudo por perguntas do paciente e respostas/informações do médico (8\%).

Em Ohio, EUA, Flocke, Miller et al. ${ }^{8}$ conduziram estudo transversal, com 2.881 pacientes de 138 médicos de família e comunidade de 84 unidades de APS, que mostrou, após análise qualitativa e quantitativa do estilo de interação médico-paciente nas consultas, que $49 \%$ deles faziam uma abordagem classificada como "focada na pessoa" (buscavam a "agenda" dos pacientes e negociavam o manejo dos problemas com os pacientes), 20\% como "biomédica" (centrados na doença, faziam poucas perguntas de aspetos psicossociais e pouco negociavam o manejo dos problemas com os pacientes), $16 \%$ como "biopsicossocial" (eram mais centrados na doença, mas faziam perguntas de aspectos psicossociais) e 14\% como de "alto controle médico" (dominavam a consulta e desconsideravam a "agenda" dos pacientes).

Roy, Vanheule et al. ${ }^{9}$, em pesquisa qualitativa, com o objetivo de conhecer os entendimentos dos médicos de família e comunidade acerca da sua prática, analisaram, por meio de entrevistas semiestruturadas, as narrativas de 19 médicos belgas sobre as suas percepções a respeito do que seriam consultas boas e más. Foram caracterizados, ao final, os seguintes tipos de discurso: o biomédico, o centrado na comunicação com o paciente, o focado na resolução de problemas e o orientado para a satisfação do paciente, mostrando não existir uma forma uniforme pela qual os médicos de família e comunidade percebem a sua prática.

É nesse contexto que o presente artigo tem como objetivo identificar as características presentes no profissional médico que se associam com o alto grau de orientação ao MCCP atribuído pelos pacientes a esse profissional. Espera-se que esse conhecimento possa auxiliar no ensino e no estímulo ao uso do MCCP pelos profissionais médicos. 


\section{Metodologia}

Este é um estudo transversal realizado com pacientes hipertensos e/ou diabéticos e seus respectivos médicos, em 12 unidades de um serviço de APS de Porto Alegre, Rio Grande do Sul, Brasil, entre novembro de 2013 e novembro de 2014.

O referido serviço pertence a um hospital público federal e é responsável por uma população de aproximadamente 120 mil pessoas. Suas equipes de APS são multiprofissionais e heterogêneas com relação ao número e à categoria profissional, sendo compostas por médicos de família e comunidade, enfermeiros e agentes comunitários de saúde, além de psicólogos, assistentes sociais, nutricionistas, terapeutas ocupacionais, farmacêuticos e cirurgiões dentistas. Nesse serviço são desenvolvidos os programas de Residência em Medicina de Família e Comunidade, desde 1980 (um dos mais antigos formadores de profissionais para APS do país), e em Residência Integrada em Saúde (para outras categorias profissionais), desde 2004.

A escolha por se trabalhar com pacientes hipertensos e/ou diabéticos se deve ao fato de esses pacientes, por tratarem doenças crônicas, se exporem de forma mais sistemática ao cuidado longitudinal de seus médicos. Além disso, a hipertensão arterial sistêmica (HAS) e o diabetes mellitus (DM) são problemas de saúde prevalentes, transcendentes e suscetíveis às ações desenvolvidas em APS $^{10-13}$.

Foram incluídos no estudo os pacientes maiores de 18 anos, inscritos no Programa de HAS e DM (Hiperdia) da respectiva unidade de saúde, com consulta médica marcada para a semana seguinte e duas ou mais consultas realizadas nos últimos 18 meses com o mesmo médico da consulta agendada. Esse último critério teve como finalidade assegurar um vínculo mínimo entre o médico e o paciente atendido. Foram excluídos os pacientes sem capacidade física e/ ou mental para responderem ao questionário $\mathrm{e}$ aqueles portadores de DM tipo I. Todos os médicos que tiveram um ou mais pacientes incluídos foram entrevistados.

Com relação aos pacientes, entrevistou-se 34 por unidade de saúde $(\mathrm{n}=408)$. Os critérios de elegibilidade foram verificados por meio da revisão dos prontuários dos pacientes, cadastrados no Hiperdia, com consulta médica marcada para a semana seguinte. As entrevistas com os pacientes ocorreram na unidade de saúde, logo após a consulta do paciente com o médico. Foram incluídas as 34 consultas consecutivas (em sequência) de cada unidade de saúde presentes nas agendas médicas marcadas para a semana seguinte da revisão das agendas e prontuários e que preencheram os critérios de inclusão - independentemente do médico, do dia da semana ou do turno de marcação (manhã ou tarde). As faltas dos pacientes às consultas $(n=99)$ e as recusas $(n=32)$ foram repostas em consultas consecutivas, seguindo-se o agendamento. As entrevistas com os médicos ocorreram após a coleta com os pacientes ter sido finalizada em cada unidade de saúde. Todos os 53 médicos que tiveram um ou mais pacientes entrevistados responderam ao questionário (de um total de 63 médicos do serviço).

O grau de orientação ao MCCP foi medido pelo instrumento "Percepção do paciente sobre o centramento da consulta" (PPCC). Elaborado por Stewart et al. ${ }^{14}$, o PPCC é constituído por 14 questões. As quatro primeiras são referentes à medida do primeiro componente do MCCP, "explorando a saúde, a doença e a experiência da doença"; as nove seguintes (da quinta até a décima-terceira questão do instrumento), ao terceiro, "elaborando um plano conjunto de manejo dos problemas", e a última questão, ao segundo componente, "entendendo a pessoa como um todo". Para as respostas, utiliza-se uma escala Likert, graduada em quatro itens. A média aritmética das respostas atribuídas às 14 questões é igual ao escore geral do PPCC. Da mesma forma, a média aritmética das respostas atribuídas às quatro primeiras questões é igual ao escore do primeiro componente; a média aritmética das respostas conferidas às nove perguntas seguintes (da quinta até a décima-terceira) é igual ao escore do terceiro componente, $\mathrm{e}$ a resposta indicada para o décimo-quarto item é igual ao escore do segundo componente. O PPCC foi validado no Brasil por Kolling e Harzheim ${ }^{15}$. O questionário, aplicado aos médicos, coletou as seguintes informações: idade, tempo de formado, formação acadêmica, especialidade, tempo de trabalho no serviço e na unidade e se ele já havia participado de alguma educação continuada a respeito da abordagem centrada na pessoa ou do MCCP no serviço de APS estudado ou fora dele (aqui compreendida como um espaço de formação que visa o aperfeiçoamento profissional do médico, oferecido pelo serviço de saúde e que pode utilizar quaisquer metodologias).

A revisão dos prontuários foi feita pelo coordenador de campo, sendo esse o responsável pela inclusão dos pacientes no estudo, e as entrevistas, por nove entrevistadores treinados. Para a coleta de dados, utilizaram-se netbooks e o software Le Sphinx Plus. 
As análises estatísticas foram realizadas com nível de significância de 5\%, bicaudal. Os dados são apresentados por meio de médias e desvios -padrão, de medianas e intervalos interquartílicos, de proporções e de respectivos intervalos de confiança. A medida da percepção do grau de orientação ao MCCP foi calculado por meio de escores em uma escala de 1 (que equivale à mais alta orientação ao MCCP) a 4 (que equivale à mais baixa orientação ao MCCP). Com relação à estimativa dos escores de cada médico, as análises foram ponderadas pelo número de pacientes entrevistados de cada profissional. Utilizou-se a mediana como ponto de corte entre a mais alta e a mais baixa orientação ao MCCP, tendo sido estimada as medianas, com respectivo intervalo interquartílico, dos escores dos componentes 1 , 2, 3 e geral de cada médico. Dessa forma, o baixo escore geral do PPCC (para valores pertencentes ao intervalo entre 1 e a respectiva mediana) corresponde a alta orientação ao MCCP e o alto escore geral do PPCC (para valores pertencentes ao intervalo entre a respectiva mediana e 4) a baixa orientação ao MCCP.

Além disso, para a identificação das variáveis que se associam ao alto escore do PPCC, utilizouse, pelo fato de os dados não satisfazerem as exigências dos métodos clássicos, testes não paramétricos: coeficiente de correlação de Spearman, Kruskal-Wallis e Mann-Whitney U. Essas análises foram realizadas no IBM SPSS Statistics 18 .

Este projeto foi aprovado pelos comitês de ética das instituições envolvidas no estudo (número de protocolo 08147413.1.0000.5347). Foram realizadas entrevistas apenas com pacientes e médicos que aceitaram participar e assinaram o Termo de Consentimento Livre e Esclarecido.

\section{Resultados}

A Tabela 1 apresenta o perfil dos médicos segundo variáveis sociodemográficas e relativas à formação acadêmica, ao tempo de trabalho e à participação em educação continuada relativa à abordagem centrada na pessoa ou ao MCCP. Observa-se que metade da amostra tem entre 31 e 50 anos (com média de 49,2), e também que aproximadamente metade é homem $(52,8 \%)$. Todos os entrevistados são brancos. A maioria possui 11 anos ou mais de formado $(88,7 \%)$ e a quase totalidade é especialista em MFC (o profissional sem formação em MFC referiu ter concluído residência em pediatria). Com relação ao tempo de trabalho, $64,2 \%$ e $56,6 \%$ têm 11 anos ou mais
Tabela 1. Características da amostra de médicos segundo variáveis sociodemográficas, acadêmicas e ocupacionais de um serviço de APS de Porto Alegre, Rio Grande do Sul, Brasil, 2015.

\begin{tabular}{|c|c|c|}
\hline Variáveis & n (\%) & $\begin{array}{l}\text { média } \\
\text { (DP) }\end{array}$ \\
\hline Idade & & $49,2(9,6)$ \\
\hline 31 a 40 anos & $13(24,5)$ & \\
\hline 41 a 50 anos & $12(22,6)$ & \\
\hline 51 a 60 anos & $24(45,3)$ & \\
\hline$>60$ anos & $4(7,5)$ & \\
\hline Sexo masculino & $28(52,8)$ & \\
\hline Cor branca & $53(100)$ & \\
\hline Tempo de formado (em anos) & & $23,2(9,0)$ \\
\hline 6 a 10 anos & $6(11,3)$ & \\
\hline 11 a 20 anos & $13(24,5)$ & \\
\hline 21 a 30 anos & $21(39,6)$ & \\
\hline$>30$ anos & $13(24,5)$ & \\
\hline $\begin{array}{l}\text { Possui residência ou prova de } \\
\text { título em Medicina de Família } \\
\text { e Comunidade }\end{array}$ & $52(98,1)$ & \\
\hline $\begin{array}{l}\text { Tempo de trabalho no serviço } \\
\text { (em anos) }\end{array}$ & & $15,7(9,6)$ \\
\hline Até 5 anos & $11(20,7)$ & \\
\hline 6 a 10 anos & $8(15,1)$ & \\
\hline 11 a 20 anos & $10(18,9)$ & \\
\hline 21 a 30 anos & $24(45,3)$ & \\
\hline $\begin{array}{l}\text { Tempo de trabalho na unidade } \\
\text { (em anos) }\end{array}$ & & $13,6(9,3)$ \\
\hline Até 5 anos & $15(28,3)$ & \\
\hline 6 a 10 anos & $8(15,1)$ & \\
\hline 11 a 20 anos & $12(22,6)$ & \\
\hline 21 a 30 anos & $18(34,0)$ & \\
\hline $\begin{array}{l}\text { Você já participou de alguma } \\
\text { educação continuada em ACP } \\
\text { ou MCCP neste serviço? }\end{array}$ & & \\
\hline Sim & $31(58,5)$ & \\
\hline $\begin{array}{l}\text { Você já participou de alguma } \\
\text { educação continuada em ACP } \\
\text { ou MCCP fora deste serviço? }\end{array}$ & & \\
\hline Sim & $19(35,8)$ & \\
\hline
\end{tabular}

$\mathrm{DP}=$ desvio padrão. $\mathrm{ACP}=$ abordagem centrada na pessoa. MCCP = método clínico centrado na pessoa.

Fonte: Autores

de trabalho no serviço e na unidade de saúde, respectivamente. A respeito de participação em educação continuada relativa à abordagem centrada na pessoa ou ao MCCP, $58,5 \%$ e $35,8 \%$, respectivamente, referiram participação em atividade no serviço de APS estudado e fora dele.

$\mathrm{Na}$ Tabela 2, pode-se observar o resultado dos escores calculados para cada médico por meio 
das medianas e dos intervalos interquartílicos da variável mediana dos escores dos componentes do PPCC e do escore geral estimados para cada profissional, respectivamente.

As variáveis contínuas (idade, tempo de formado, tempo no serviço e tempo na unidade) e categóricas (unidade de saúde, sexo, participação em educação continuada no serviço de APS estudado ou fora dele) associadas com o baixo escore dos componentes do PPCC e o escore geral (o que corresponde a alta orientação ao MCCP) são apresentadas nas tabelas 3 e 4, respectivamente. Todas as variáveis contínuas são correlacionadas com o escore do componente 3 ("elaborando um plano conjunto de manejo dos problemas”) e com o escore geral, porém negativamente, ou seja, quanto maior, por exemplo, o tempo de trabalho na unidade, menor a mediana do escore geral (o que significa maior grau de orientação ao MCCP). Destaca-se que todas as correlações apresentam coeficientes de até 0,29 , considerados de intensidade fraca, porém com significância estatística. As variáveis idade, tempo de formado, tempo no serviço e tempo na unidade também se mostraram correlacionadas entre si, com coeficientes de correlação positivos e estatisticamente significativos em todas as correlações, com todos os coeficientes maiores do que 0,6 , considerados de intensidade forte.

$\mathrm{Na}$ Tabela 4, observa-se que as medianas dos escores médios dos componentes 2 ("entendendo a pessoa como um todo"), 3 ("elaborando um plano conjunto de manejo dos problemas”)

Tabela 2. Medida de tendência central e variabilidade dos escores do instrumento "Percepção do Paciente sobre o Centramento da Consulta" (PPCC) calculados por médico*. Estudo em um serviço de APS de Porto Alegre, Rio Grande do Sul, Brasil, 2015.

\begin{tabular}{|c|c|c|}
\hline Escore $(1-4)$ & mediana & IIQ \\
\hline \multicolumn{3}{|l|}{ Escore do primeiro } \\
\hline componente do PPCC & 1,00 & $1,00-1,25$ \\
\hline \multicolumn{3}{|l|}{ Escore do segundo } \\
\hline componente do PPCC & 1,00 & $1,00-1,00$ \\
\hline \multicolumn{3}{|l|}{ Escore do terceiro } \\
\hline componente do PPCC & 1,28 & $1,17-1,44$ \\
\hline Escore geral & 1,21 & $1,14-1,36$ \\
\hline \multicolumn{3}{|c|}{$\begin{array}{l}\text { IIQ = intervalo interquartílico. PPCC = percepção do paciente } \\
\text { sobre o centramento da consulta. } \\
\text { * São apresentadas as medianas e os IIQ da variável mediana } \\
\text { dos escores dos componentes e do escore geral do PPCC } \\
\text { calculados para cada médico. }\end{array}$} \\
\hline
\end{tabular}

e geral atribuídos às médicas foram significativamente menores do que as dos médicos, indicando que elas desempenham em maior grau o MCCP. Além disso, destaca-se que a participação em educação continuada acerca do tema esteve associada ao maior grau de orientação à pessoa. No entanto, essa associação foi mais significativa em relação à participação em educação continuada fora do serviço. A mediana do escore médio do componente 3 ("elaborando um plano conjunto de manejo dos problemas") atribuído aos profissionais que referiram ter participado de educação continuada no próprio serviço foi significativamente menor do que a dos que não referiram, demonstrando que ter participado de educação continuada no próprio serviço aumenta o grau de orientação a esse componente.

Da mesma forma, as medianas dos escores médios do componente 2 ("entendendo a pessoa como um todo"), 3 ("elaborando um plano conjunto de manejo dos problemas") e geral atribuídos aos profissionais que referiram ter participado de educação continuada fora do serviço foram significativamente menores do que as dos que não referiram, evidenciando que a participação em educação continuada fora do serviço aumenta o grau de orientação ao MCCP. Nota-se que as análises envolvendo o componente 1 ("explorando a saúde, a doença e a experiência da doença") não se mostraram significativas, o que permite concluir que esse componente não é influenciado por características presentes no profissional médico estudadas na presente pesquisa.

Quando se buscou verificar a significância das diferenças entre as medianas dos escores médios dos componentes 1 ("explorando a saúde, a doença e a experiência da doença"), 2 ("entendendo a pessoa como um todo"), 3 ("elaborando um plano conjunto de manejo dos problemas") e geral calculados por unidade de saúde, encontrou-se diferença significativa entre as medianas em todos eles. Esses achados revelam que existe diferença significativa entre o grau de orientação ao MCCP entre as unidades de saúde do serviço de APS estudado.

\section{Discussão}

Os resultados apresentados evidenciam que características demográficas e relativas à experiência profissional e à participação em educação continuada dos médicos influenciam o desempenho da abordagem clínica desses profissionais. A correlação das variáveis idade, tempo de forma- 
Tabela 3. Análise da associação entre as variáveis "idade", "tempo de formado", "tempo de trabalho no serviço" e "na unidade" com o baixo escore dos componentes e do escore geral do instrumento "Percepção do Paciente sobre o Centramento da Consulta” (o que corresponde à alta orientação ao Método Clínico Centrado na Pessoa). Estudo em um serviço de APS de Porto Alegre, Rio Grande do Sul, Brasil, 2015.

\begin{tabular}{lcccc}
\hline \multirow{2}{*}{ Variáveis contínuas } & \multicolumn{4}{c}{ Mediana dos escores atribuídos ao médico } \\
\cline { 2 - 5 } & $\begin{array}{c}\text { Componente } \mathbf{1} \\
\mathbf{r}_{\mathrm{s}} \text { (valor-p) }\end{array}$ & $\begin{array}{c}\text { Componente } 2 \\
\mathbf{r}_{\mathrm{s}} \text { (valor-p) }\end{array}$ & $\begin{array}{c}\text { Componente 3 } \\
\mathbf{r}_{\mathrm{s}} \text { (valor-p) }\end{array}$ & $\begin{array}{c}\text { Escore geral } \\
\mathbf{r}_{\mathrm{s}} \text { (valor-p) }\end{array}$ \\
\hline $\begin{array}{l}\text { Idade (em anos) } \\
\begin{array}{l}\text { Tempo de formado (em anos) } \\
\text { Tempo de trabalho no serviço }\end{array}\end{array}$ & $\mathrm{NS}$ & $\mathrm{NS}$ & $-0,19(<0,001)$ & $-0,24(<0,001)$ \\
$\begin{array}{l}\text { (em anos) } \\
\text { Tempo de trabalho na unidade }\end{array}$ & $\mathrm{NS}$ & $-0,14(0,004)$ & $-0,25(<0,001)$ & $-0,29(<0,001)$ \\
$(\mathrm{em}$ anos) & $\mathrm{NS}$ & $-0,11(0,023)$ & $-0,15 \quad(0,003)$ & $-0,26(<0,001)$ \\
\hline
\end{tabular}

NS = não significativo. ${ }^{*}$ Coeficiente de correlação de Spearman.

Fonte: Autores.

Tabela 4. Análise da associação entre as variáveis "unidade de saúde", "sexo" e "participação em educação continuada" com o baixo escore dos componentes e do escore geral do instrumento "Percepção do Paciente sobre o Centramento da Consulta" (o que corresponde à alta orientação ao Método Clínico Centrado na Pessoa). Estudo em um serviço de APS de Porto Alegre, Rio Grande do Sul, Brasil, 2015.

\begin{tabular}{|c|c|c|c|c|}
\hline \multirow[b]{2}{*}{ Variáveis categóricas } & \multicolumn{4}{|c|}{ Medianas dos escores ${ }^{\star}$} \\
\hline & $\begin{array}{c}\text { Componente } 1 \\
\text { mediana } \\
(\text { IIQ }) \\
(\text { valor-p })^{\star *}\end{array}$ & $\begin{array}{c}\text { Componente } 2 \\
\text { mediana } \\
(\text { IIQ }) \\
(\text { valor-p) })^{\star *}\end{array}$ & $\begin{array}{c}\text { Componente } 3 \\
\text { mediana } \\
(\text { IIQ }) \\
(\text { valor-p })^{\star *}\end{array}$ & $\begin{array}{c}\text { Escore geral } \\
\text { mediana } \\
(\text { IIQ }) \\
(\text { valor-p) })^{* *}\end{array}$ \\
\hline \multicolumn{5}{|l|}{ Unidade de saúde } \\
\hline A & $\begin{array}{c}1,00 \\
(1,00-1,25)\end{array}$ & $\begin{array}{c}1,00 \\
(1,00-1,00)\end{array}$ & $\begin{array}{c}1,11 \\
(1,11-1,22)\end{array}$ & $\begin{array}{c}1,14 \\
(1,14-1,29)\end{array}$ \\
\hline B & $\begin{array}{c}1,00 \\
(1,00-1,25)\end{array}$ & $\begin{array}{c}1,00 \\
(1,00-1,00)\end{array}$ & $\begin{array}{c}1,11 \\
(1,11-1,28)\end{array}$ & $\begin{array}{c}1,07 \\
(1,07-1,25)\end{array}$ \\
\hline $\mathrm{C}$ & $\begin{array}{c}1,13 \\
(1,00-1,25)\end{array}$ & $\begin{array}{c}1,00 \\
(1,00-1,00)\end{array}$ & $\begin{array}{c}1,33 \\
(1,22-1,44)\end{array}$ & $\begin{array}{c}1,30 \\
(1,21-1,43)\end{array}$ \\
\hline $\mathrm{D}$ & $\begin{array}{c}1,00 \\
(1,00-1,13)\end{array}$ & $\begin{array}{c}1,00 \\
(1,00-1,00)\end{array}$ & $\begin{array}{c}1,22 \\
(1,22-1,61)\end{array}$ & $\begin{array}{c}1,14 \\
(1,14-1,50)\end{array}$ \\
\hline $\mathrm{E}$ & $\begin{array}{c}1,00 \\
(1,00-1,50)\end{array}$ & $\begin{array}{c}1,00 \\
(1,00-1,00)\end{array}$ & $\begin{array}{c}1,33 \\
(1,22-1,33)\end{array}$ & $\begin{array}{c}1,21 \\
(1,14-1,36)\end{array}$ \\
\hline $\mathrm{F}$ & $\begin{array}{c}1,00 \\
(1,00-1,25)\end{array}$ & $\begin{array}{c}1,00 \\
(1,00-1,50)\end{array}$ & $\begin{array}{c}1,33 \\
(1,17-1,50)\end{array}$ & $\begin{array}{c}1,29 \\
(1,14-1,43)\end{array}$ \\
\hline G & $\begin{array}{c}1,13 \\
(1,00-1,50)\end{array}$ & $\begin{array}{c}1,00 \\
(1,00-1,00)\end{array}$ & $\begin{array}{c}1,22 \\
(1,11-1,39)\end{array}$ & $\begin{array}{c}1,29 \\
(1,21-1,29)\end{array}$ \\
\hline $\mathrm{H}$ & $\begin{array}{c}1,13 \\
(1,00-1,25)\end{array}$ & $\begin{array}{c}1,00 \\
(1,00-1,00)\end{array}$ & $\begin{array}{c}1,44 \\
(1,11-1,44)\end{array}$ & $\begin{array}{c}1,29 \\
(1,14-1,39)\end{array}$ \\
\hline I & $\begin{array}{c}1,07 \\
(1,00-1,38)\end{array}$ & $\begin{array}{c}1,00 \\
(1,00-1,00)\end{array}$ & $\begin{array}{c}1,33 \\
(1,33-1,39)\end{array}$ & $\begin{array}{c}1,21 \\
(1,21-1,36)\end{array}$ \\
\hline $\mathrm{J}$ & $\begin{array}{c}1,00 \\
(1,00-1,25)\end{array}$ & $\begin{array}{c}1,00 \\
(1,00-1,00)\end{array}$ & $\begin{array}{c}1,22 \\
(1,22-1,61)\end{array}$ & $\begin{array}{c}1,14 \\
(1,14-1,43)\end{array}$ \\
\hline $\mathrm{K}$ & $\begin{array}{c}1,00 \\
(1,00-1,00)\end{array}$ & $\begin{array}{c}1,00 \\
(1,00-1,00)\end{array}$ & $\begin{array}{c}1,33 \\
(1,11-1,33)\end{array}$ & $\begin{array}{c}1,36 \\
(1,14-1,36)\end{array}$ \\
\hline $\mathrm{L}$ & $\begin{array}{c}1,25 \\
(1,00-1,25) \\
(0,002)^{\#}\end{array}$ & $\begin{array}{c}1,00 \\
(1,00-1,00) \\
(<0,001)^{\# \#}\end{array}$ & $\begin{array}{c}1,56 \\
(1,33-1,56) \\
(<0,001)^{\# \# \#}\end{array}$ & $\begin{array}{c}1,36 \\
(1,36-1,36) \\
(<0,001)\end{array}$ \\
\hline
\end{tabular}


Tabela 4. Análise da associação entre as variáveis “unidade de saúde”, "sexo" e "participação em educação continuada" com o baixo escore dos componentes e do escore geral do instrumento "Percepção do Paciente sobre o Centramento da Consulta" (o que corresponde à alta orientação ao Método Clínico Centrado na Pessoa). Estudo em um serviço de APS de Porto Alegre, Rio Grande do Sul, Brasil, 2015.

\begin{tabular}{|c|c|c|c|c|}
\hline \multirow[b]{2}{*}{ Variáveis categóricas } & \multicolumn{4}{|c|}{ Medianas dos escores $^{*}$} \\
\hline & $\begin{array}{c}\text { Componente } 1 \\
\text { mediana } \\
(\text { IIQ }) \\
\left(\text { valor-p) }^{* *}\right.\end{array}$ & $\begin{array}{c}\text { Componente } 2 \\
\text { mediana } \\
(\text { IIQ }) \\
(\text { valor-p })^{\star *}\end{array}$ & $\begin{array}{c}\text { Componente } 3 \\
\text { mediana } \\
(\text { IIQ }) \\
(\text { valor-p) })^{* *}\end{array}$ & $\begin{array}{c}\text { Escore geral } \\
\text { mediana } \\
(\text { IIQ }) \\
(\text { valor-p })^{* *}\end{array}$ \\
\hline \multicolumn{5}{|l|}{ Sexo } \\
\hline Masculino & $\begin{array}{c}1,00 \\
(1,00-1,25)\end{array}$ & $\begin{array}{c}1,00 \\
(1,00-1,00)\end{array}$ & $\begin{array}{c}1,33 \\
(1,22-1,44)\end{array}$ & $\begin{array}{c}1,29 \\
(1,21-1,36)\end{array}$ \\
\hline Feminino & $\begin{array}{c}1,00 \\
(1,00-1,25) \\
(0,157)\end{array}$ & $\begin{array}{c}1,00 \\
(1,00-1,00) \\
(<0,001)\end{array}$ & $\begin{array}{c}1,22 \\
(1,17-1,33) \\
(<0,001)\end{array}$ & $\begin{array}{c}1,21 \\
(1,14-1,36) \\
(<0,001)\end{array}$ \\
\hline \multicolumn{5}{|l|}{$\begin{array}{l}\text { Participação em educação } \\
\text { continuada no serviço }\end{array}$} \\
\hline $\operatorname{sim}$ & $\begin{array}{c}1,13 \\
(1,00-1,25)\end{array}$ & $\begin{array}{c}1,00 \\
(1,00-1,00)\end{array}$ & $\begin{array}{c}1,28 \\
(1,17-1,39)\end{array}$ & $\begin{array}{c}1,21 \\
(1,14-1,36)\end{array}$ \\
\hline não & $\begin{array}{c}1,13 \\
(1,00-1,25) \\
(0,458)\end{array}$ & $\begin{array}{c}1,00 \\
(1,00-1,00) \\
(0,090)\end{array}$ & $\begin{array}{c}1,33 \\
(1,22-1,56) \\
(0,018)\end{array}$ & $\begin{array}{c}1,36 \\
(1,14-1,43) \\
(0,376)\end{array}$ \\
\hline \multicolumn{5}{|l|}{$\begin{array}{l}\text { Participação em educação } \\
\text { continuada fora do serviço }\end{array}$} \\
\hline $\operatorname{sim}$ & $\begin{array}{c}1,00 \\
(1,00-1,25)\end{array}$ & $\begin{array}{c}1,00 \\
(1,00-1,00)\end{array}$ & $\begin{array}{c}1,22 \\
(1,11-1,39)\end{array}$ & $\begin{array}{c}1,21 \\
(1,14-1,29)\end{array}$ \\
\hline não & $\begin{array}{c}1,00 \\
(1,00-1,25) \\
(0,059)\end{array}$ & $\begin{array}{c}1,00 \\
(1,00-1,00) \\
(0,003)\end{array}$ & $\begin{array}{c}1,33 \\
(1,17-1,44) \\
(0,001)\end{array}$ & $\begin{array}{c}1,25 \\
(1,14-1,39) \\
(0,002)\end{array}$ \\
\hline \multicolumn{5}{|c|}{ 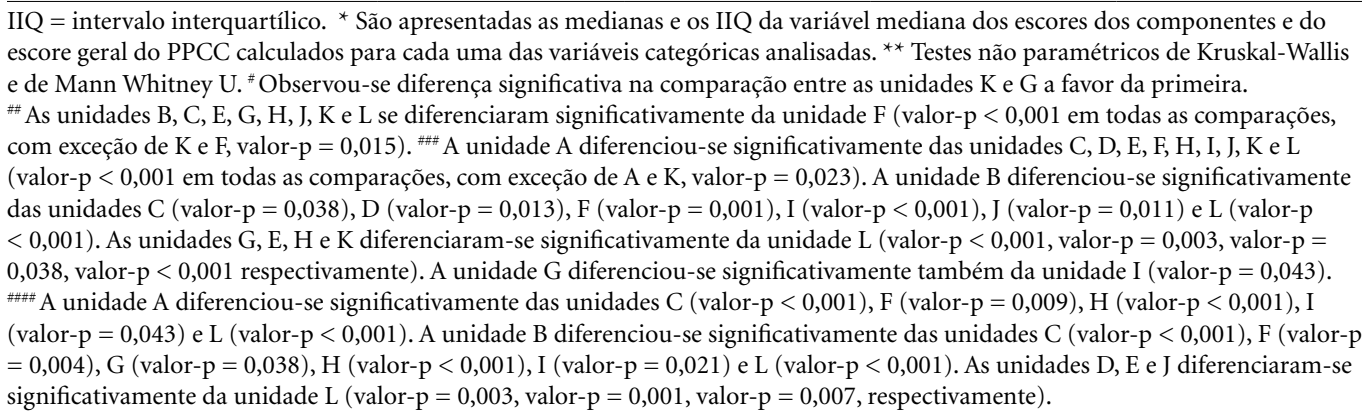 } \\
\hline
\end{tabular}

Fonte: Autores

do, tempo no serviço e na unidade com as medianas dos escores do componente 3 ("elaborando um plano conjunto de manejo dos problemas") e geral atribuídos pelos pacientes atendidos por cada médico, de forma que o aumento daquelas varia com a diminuição das medianas (o que significa maior orientação ao MCCP), destaca a importância da experiência de vida e profissional na modelagem da abordagem do médico. De fato, o tempo na unidade de saúde favorece o maior co- nhecimento das pessoas, famílias e comunidade, permitindo assim que o MCCP seja mais bem utilizado.

De acordo com achados referentes à variável sexo, pode-se concluir que as médicas são significativamente mais centradas nas pessoas atendidas por elas do que os médicos. A diferença de abordagem entre os profissionais do sexo masculino e feminino foi investigada pela pesquisa de Law e Britten ${ }^{16}$. Nesse estudo inglês, foram ana- 
lisadas consultas gravadas em áudio de 41 médicos de família e comunidade, selecionados de um grupo de voluntários, e demonstrou-se que os profissionais do sexo feminino tinham maiores escores de centramento no paciente do que os do sexo masculino. Além disso, mostrou-se que os mais altos escores de centramento no paciente foram atribuídos às interações entre médicas e pacientes mulheres, não se encontrando diferença entre os escores observados nas consultas médicas-pacientes homens e médicos-pacientes homens, e que as mais baixas médias foram verificadas entre médicos e pacientes mulheres.

Por outro lado, Bertakis, Franks et al. ${ }^{17}$, em análise de consultas médicas em APS, em Rochester, EUA, gravadas em vídeo ( $\mathrm{n}=100$, de um total de 594 elegíveis), por meio do instrumento Medida da Comunicação Centrada no Paciente (IMCCP), não encontraram diferença entre o escore geral de IMCCP entre os dois sexos, refletindo efeitos opostos para o primeiro componente ("explorando a saúde, a doença e a experiência da doença"), que foi maior para médicos do sexo feminino, e para o segundo ("entendendo a pessoa como um todo"), que foi menor para médicos do sexo feminino. O terceiro componente ("elaborando um plano conjunto de manejo dos problemas") não foi associado com o sexo. Da mesma forma, a concordância de gênero também não foi associada ao escore geral do IMCCP.

Com relação à participação em educação continuada, aqueles profissionais que referiram participação em atividade fora do serviço foram significativamente mais centrados nas pessoas por eles atendidas do que os que não realizaram esse tipo de atividade. Esse dado foi mais consistente do que aquele captado pela participação ou não em educação continuada dentro do próprio serviço (que só mostrou significância estatística nas análises do terceiro componente). Essas diferenças levantam dois importantes pontos a serem aprofundados. Primeiro, a educação continuada exerce um efeito positivo sobre a abordagem do médico. Esse achado corrobora as evidências apontadas na literatura. Como se pode depreender de duas revisões sistemáticas ${ }^{18,19} \mathrm{e}$ de ensaios clínicos a respeito do tema, a prática centrada na pessoa pode ser aprendida por meio de treinamento em habilidades de comunicação $\mathrm{o}^{20-26} \mathrm{e}$ as competências adquiridas pelos médicos após o treinamento podem afetar positivamente os resultados de saúde dos pacientes ${ }^{20-23}$. Segundo, o interesse do profissional sobre o tema pode ser uma variável fundamental para a análise do su- cesso ou não de determinada atividade educativa. Essa hipótese ganha força à medida em que consideramos a busca por educação continuada fora do serviço como um indicador do interesse do profissional a respeito do tema.

Um resultado importante deste estudo foi o de reconhecer a diferença significativa entre o grau de orientação ao MCCP praticado entre as unidades de saúde do serviço de APS estudado. Esse dado sustenta que o contexto no qual cada médico trabalha incide sobre sua prática. Aita e colaboradores $^{6}$ - em estudo qualitativo que analisou o cuidado centrado no paciente em uma amostra aleatória de consultas de 44 médicos em 18 unidades de APS de Nebraska, EUA, e que envolveu variadas técnicas de coleta de dados e a imersão dos pesquisadores por um período de um a dois meses em cada unidade-identificaram os seguintes conjuntos de fatores influenciadores da interação médico-paciente: 1) características do médico (estilo, valores e filosofia da medicina), 2) características do paciente (prioridades, valores e filosofia da saúde), 3) características das unidades de APS (organização, prioridades e cultura e sua relação com a filosofia do médico) e 4) características da comunidade (cultura, prioridades e sua relação com as expectativas dos cuidados médicos). Da mesma forma, Mead e Bowert ${ }^{4} \mathrm{e}$ Willems et al. ${ }^{5}$, em seus trabalhos de revisão, concordam com a influência do contexto sobre a prática.

Podemos reconhecer limitações no presente estudo. Inicialmente, existe a possibilidade de o tamanho amostral ter influenciado negativamente o poder das análises. O instrumento PPCC, por sua vez, também apresenta limitações, sendo a maior, talvez, a que envolve a medida do componente 2 ("entendendo a pessoa como um todo") do MCCP, pois para isso é usada apenas uma questão geral. Além disso, esta é uma pesquisa avaliativa e transversal, sujeita às limitações próprias desse tipo de delineamento. Uma delas é a validade externa, já que seus resultados devem ser discutidos mediante a realidade local dos serviços. Por último, esse estudo foi realizado em diferentes unidades de saúde de um mesmo serviço de APS (vinculado a um hospital federal e com longa história de formação de recursos humanos para a APS), sendo importante, portanto, em pesquisas futuras, a inclusão de outros serviços, a fim de que se possa estimar também os efeitos do serviço de APS sobre as características da abordagem desempenhada por seus profissionais médicos. 


\section{Considerações finais}

Os resultados apresentados neste estudo trazem importantes implicações para a compreensão das características presentes no profissional médico que influenciam sua abordagem, e também para a pesquisa e formação profissional na área.

Embora a presente pesquisa tenha demonstrado a influência positiva do aumento da idade, do tempo de formado e de trabalho sobre a aplicação da abordagem centrada na pessoa, estudos futuros devem identificar os elementos específicos que, agregados pelo médico ao longo do tempo, fazem com que o profissional se torne mais centrado nas pessoas por ele atendidas. Da mesma forma, pode-se estender essa questão para o âmbito das diferenças encontradas entre os profissionais do sexo masculino e feminino e entre as unidades de saúde. Ou seja, quais os elementos específicos que explicam a maior orientação às pessoas pelas médicas, bem como as diferenças entre as unidades de saúde? São temas que merecem aprofundamento em investigações posteriores.

Acerca da formação profissional, confirma-se o efeito potencialmente modificador da abordagem médica pela educação continuada. Entretanto, em se reconhecendo o interesse do profissional a respeito do tema como uma variável que implica a produção desse efeito, faz-se necessária a criação de metodologias de educação continuada que possam justamente trabalhar com esse interesse. Especial atenção deve ser dada também à abordagem do componente 1 ("explorando a saúde, a doença e a experiência da doença") em atividades educacionais (visto que as análises que envolvem esse componente não se mostraram significativas), de modo a repensar a maneira de se ajudar os médicos a explorarem tanto a doença como a experiência da doença nas pessoas por eles atendidas.

\section{Colaboradores}

RCL Castro trabalhou na concepção do estudo, revisão da literatura, coordenação do trabalho de campo, construção e revisão da base de dados, análise e interpretação dos resultados, construção das tabelas, redação do texto e aprovação da versão a ser publicada. DR Knauth trabalhou na concepção do estudo, análise e interpretação dos resultados, revisão crítica do texto e aprovação da versão a ser publicada. 


\section{Referências}

1. Klinkman M, Weel CV. Prospects for person-centred diagnosis in general medicine. J Eval Clin Pract 2011; 17(2):365-370.

2. Weel CV. Person-centred medicine in the context of primary care: a view from the World Organization of Family Doctors (Wonca). J Eval Clin Pract 2011; 17(2):337-338.

3. Stewart M, Brown JB, Weston WW, McWhinney IR, McWilliam CL, Freeman TR. Patient-centered medicine: transforming the clinical method. London: Radcliffe Publishing; 2014.

4. Mead N, Bower P. Measuring patient-centredness: a comparison of three observation-based instruments. Patient Educ Couns 2000; 39(1):71-80.

5. Willems S, Maesschalck S, Deveugele M, Derese A, Maeseneer J. Socio-economic status of the patient and doctor-patient communication: does it make a difference? Patient Educ Couns 2005; 56(2):139-146.

6. Aita V, McIlvain H, Backer E, McVea K, Crabtree B. Patient-centered care and communication in primary care practice: what is involved? Patient Educ Couns 2005; 58(3):296-304.

7. Roter DL, Stewart M, Putnam SM, Lipkin Jr M, Stiles $\mathrm{W}$, Inui TS. Communication patterns of primary care physicians. JAMA 1997; 277(4):350-356.

8. Flocke SA, Miller WL, Crabtree BF. Relationships between physician practice style, patient satisfaction, and attributes of primary care. J Fam Pract 2002; 51(10):835-840.

9. Roy KV, Vanheule S, Deveugele M. What makes up good consultations? A qualitative study of GPs' discourses. BMC Fam Pract 2013; 14:62.

10. Brasil. Ministério da Saúde (MS). Secretaria de Atenção à Saúde. Departamento de Atenção Básica. Hipertensão arterial sistêmica. Brasília: MS; 2006.

11. Brasil. Ministério da Saúde. Estratégias para o cuidado da pessoa com doença crônica: hipertensão arterial sistêmica. Brasília: MS; 2013.

12. Brasil. Ministério da Saúde (MS). Secretaria de Atenção à Saúde. Departamento de Atenção Básica. Diabetes mellitus. Brasília: MS; 2006.

13. Brasil. Ministério da Saúde (MS). Estratégias para o cuidado da pessoa com doença crônica: diabetes mellitus. Brasília: MS; 2013.

14. Stewart M, Brown JB, Donner A, McWhinney IR, Oates J, Weston WW, Jordan J. The impact of patient-centered care on outcomes. J Fam Pract 2000; 49(9):796-804.

15. Kolling MG, Harzheim E. Avaliação do método clínico centrado na pessoa: tradução e validação do patient perception of patient centeredness questionnaire [dissertação]. Porto Alegre: Universidade Federal do Rio Grande do Sul; 2012.

16. Law SA, Britten N. Factors that influence the patient centredness of a consultation. Br J Gen Pract 1995; 45(399):520-524.

17. Bertakis KD, Franks P, Epstein RM. Patient-centered communication in primary care: physician and patient gender and gender concordance. $J$ Womens Health (Larchmt) 2009; 18(4):539-545.
18. Lewin SA, Skea ZC, Entwistle V, Zwarenstein M, Dick J. Interventions for providers to promote a patient-centred approach in clinical consultations. Cochrane Database of Systematic Reviews 2001; 4:CD003267.

19. Dwamena F, Holmes-Rovner M, Gaulden CM, Jorgenson S, Sadigh G, Sikorskii A et al. Interventions for providers to promote a patient-centred approach in clinical consultations. Cochrane Database of Systematic Reviews 2012; 12:CD003267.

20. Kinmonth AL, Woodcock A, Griffin S, Spiegal N, Campbell MJ. Randomised controlled trial of patient-centred care of diabetes in general practice: impact on current wellbeing and future disease risk. The Diabetes Care From Diagnosis Research Team. BMJ 1998; 317(7167):1202-1208.

21. Woodcock AJ, Kinmonth AL, Campbell MJ, Griffin SJ, Spiegal NM. Diabetes care from diagno- sis: effects of training in patient-centred care on beliefs, attitudes and behaviour of primary care professionals. Patient Educ Couns 1999; 37(1):65-79.

22. Cooper LA, Roter DL, Carson KA, Bone LR, Larson SM, Miller ER 3rd, Barr MS, Levine DM. A randomized trial to improve patient-centered care and hypertension control in underserved primary care patients. J Gen Intern Med. 2011 Nov;26(11):1297-1304.

23. Moral RR, Alamo MM, Jurado MA, Torres LP. Effectiveness of a learner-centred training programme for primary care physicians in using a patient-centred consultation style. Fam Pract 2001; 18(1):60-63.

24. Krasner MS, Epstein RM, Beckman H, Suchman AL, Chapman B, Mooney CJ, Quill TE. Association of an educational program in mindful communication with burnout, empathy, and attitudes among primary care physicians. JAMA 2009; 302(12):1284-1293.

25. Bonvicini KA, Perlin MJ, Bylund CL, Carroll G, Rouse RA, Goldstein MG. Impact of communication training on physician expression of empathy in patient encounters. Patient Educ Couns 2009; 75(1):3-10.

26. Helitzer DL, Lanoue M, Wilson B, Hernandez BU, Warner T, Roter D. A randomized controlled trial of communication training with primary care providers to improve patient-centeredness and health risk communication. Patient Educ Couns 2011; 82(1):21-29.

Artigo apresentado em 29/09/2020

Aprovado em 26/01/2021

Versão final apresentada em 28/01/2021

Editores-chefes: Romeu Gomes, Antônio Augusto Moura da Silva 